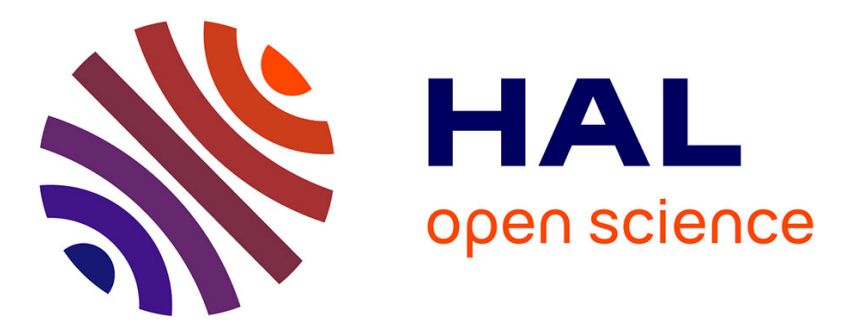

\title{
Étude de dispositifs optiques à microcavité verticale pour une émission laser polarisée
}

Christophe Levallois, Alain Le Corre, Olivier Dehaese, Hervé Folliot, Cyril

Paranthoen, Christophe Labbé, Slimane Loualiche

\section{- To cite this version:}

Christophe Levallois, Alain Le Corre, Olivier Dehaese, Hervé Folliot, Cyril Paranthoen, et al.. Étude de dispositifs optiques à microcavité verticale pour une émission laser polarisée. 9ème Colloque sur les Lasers et l'Optique Quantique, Sep 2005, Dijon, France. p. 125-126, 10.1051/jp4:2006135025. hal-00491469

\section{HAL Id: hal-00491469 \\ https://hal.science/hal-00491469}

Submitted on 11 Jun 2010

HAL is a multi-disciplinary open access archive for the deposit and dissemination of scientific research documents, whether they are published or not. The documents may come from teaching and research institutions in France or abroad, or from public or private research centers.
L'archive ouverte pluridisciplinaire HAL, est destinée au dépôt et à la diffusion de documents scientifiques de niveau recherche, publiés ou non, émanant des établissements d'enseignement et de recherche français ou étrangers, des laboratoires publics ou privés. 


\title{
Etude de dispositifs optiques à microcavité verticale pour une émission laser polarisée
}

\author{
C. Levallois, A. Le Corre, O. Dehaese, H. Folliot, C. Paranthoen, C. Labbé et S. Loualiche. \\ LENS - CNRS UMR FOTON 6082- INSA de Rennes, 20 Avenue des Buttes de Coësmes, 34043 Rennes Cedex \\ Téléphone : +33223238424 - e-mail: christophe.levallois@ens.insa-rennes.fr
}

\begin{abstract}
Résumé : Dans ce travail nous rapportons la réalisation d'un VCSEL dont la zone active est à base de puits quantiques InGaAs/InGaAsP en accord de maille sur InP. L'utilisation de cette zone active avec deux miroirs diélectriques en silicium amorphe et nitrure de silicium a permis d'obtenir un VCSEL fonctionnant à $1.55 \mu \mathrm{m}$ en pompage optique et à température ambiante. La croissance de nouvelles structures quantiques, se présentant sous forme de fils, a été réalisée en épitaxie par jets moléculaires. L'étude de ces structures quantiques semble indiquer des propriétés intéressantes pour obtenir un VCSEL stable en polarisation.
\end{abstract}

\section{1/ Introduction}

Les VCSEL émettant entre $1.3 \mu \mathrm{m}$ et $1.55 \mu \mathrm{m}$ sont potentiellement très intéressants en tant que sources émettrices pour les télécommunications optiques. Ils ont les avantages habituels reconnus aux VCSELs : possibilité de tester sur plaque les composants et facilité de couplage avec les fibres optiques. Cependant, à l'inverse des lasers à émission par la tranche, les VCSELs ne présentent aucune polarisation marquée lorsque la zone active est réalisée de façon conventionnelle sur des substrat orientés (100). La symétrie du cristal implique alors une polarisation instable pouvant changer au cours du fonctionnement du composant. Plusieurs types de solutions ont déjà été abordés pour résoudre ce problème ${ }^{1,2,3}$. Cependant, la possibilité d'introduire dans la zone active un matériau présentant une anisotropie de gain peut s'avérer intéressante puisqu'elle permettrait de fixer la polarisation sans ajouter de processus supplémentaires à la fabrication du VCSEL. ${ }^{4}$

Nous présentons dans ce résumé, la réalisation et la caractérisation d'un VCSEL à base de puits quantiques (PQs). Le succès récent de la croissance de fils quantiques (FQs) sur substrat d'InP (100) permet de mettre en cavité ces structures de façon identique à la méthode utilisée dans le cas de PQs. Les propriétés d'anisotropie très intéressantes des FQs observées en photoluminescence (PL) laissent alors entrevoir la possibilité de fixer la polarisation des VCSELs.

\section{2/ Réalisation du VCSEL}

Le faible contraste d'indice des matériaux de la filière InP rend difficile la croissance de miroirs de Bragg performants. ${ }^{5}$ Ainsi, la substitution de miroirs réalisés en épitaxie par des miroirs diélectriques permet d'obtenir des DBR (Distributed Bragg Reflectors) de haute réflectivité avec un nombre réduit de périodes. Les miroirs diélectriques utilisés sont à base de silicium amorphe $(\mathrm{a}-\mathrm{Si})$ et de nitrure de silicium $\left(\mathrm{a}-\mathrm{SiN}_{\mathrm{x}}\right)$ déposés par pulvérisation cathodique magnétron. Le différence d'indice élevée entre ces deux matériaux $(\Delta \mathrm{n}=1.9)$ à $1.55 \mu \mathrm{m}$ autorise la réalisation d'un DBR d'une réflectivité de $99.5 \%$ avec une bande spectrale de $800 \mathrm{~nm}$ (Fig. 1). L'association de tels DBR avec une zone active réalisée en MBE (Molecular Beam Epitaxy) a rendu possible le développement de VCSEL fonctionnant à $1.55 \mu \mathrm{m}$.

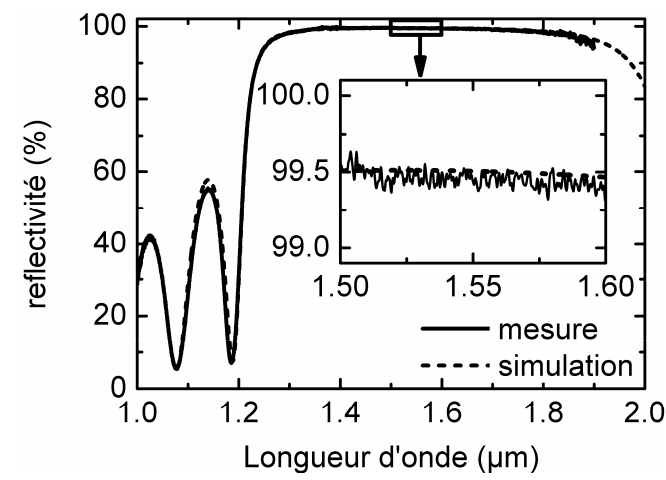

Fig. 1 : Spectre de réflectivité simulé et mesuré d'un DBR de 4,5 périodes de a-Si/a-SiN

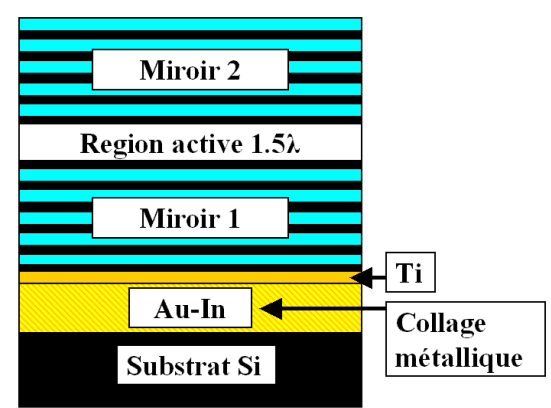

Fig 2 : Schéma final du VCSEL réalisé par collage métallique

Après la réalisation de la zone active à base de PQs (InGaAs/InGaAsP) sur substrat d'InP. Un premier DBR en $\mathrm{a}-\mathrm{Si} / \mathrm{a}-\mathrm{SiN}_{\mathrm{x}}$ est déposé pour former le miroir de fond de cavité. Cette étape est suivie par le dépôt d'un tri-couche 
métallique (Ti-Au-In). Ces couches métalliques sont utiles pour réaliser le processus de report sur un substrat hôte. En effet, la mise en contact sous une pression de $5 \mathrm{~kg} \mathrm{~cm}^{-2}$, à une température de $240{ }^{\circ} \mathrm{C}$, d'une telle structure, avec un substrat de silicium recouvert d'or, provoque la formation d'un alliage métallique Au-In permettant le report sur un substrat de silicium. ${ }^{6}$ Le retrait du substrat d'InP est réalisé par une attaque chimique sélective. La phase de fabrication du VCSEL est ensuite clôturée par le dépôt d'un second miroir diélectrique (Fig. 2).

\section{3/ Caractérisations et résultats}

Les VCSELs ainsi fabriqués sont pompés optiquement à une longueur d'onde de $1.064 \mu \mathrm{m}$. Le faisceau de pompe après réflexion sur une séparatrice est focalisé pour former un spot de $12 \mu \mathrm{m}$ de diamètre sur le DBR supérieur. Le signal émis par ce même DBR est transmis à travers la séparatrice pour être collecté par une fibre optique connectée à un analyseur de spectre. La source pulsée utilisée permet d'obtenir des pulses d'une nanoseconde avec un taux de répétition de $6,6 \mathrm{kHz}$. L'émission laser a été obtenue à température ambiante pour une puissance crête de $200 \mathrm{~mW}$. Nous avons pu observer une raie laser centrée vers 1,535 $\mu \mathrm{m}$ et tracer l'évolution de la puissance optique moyenne émise en fonction de la puissance crête de la pompe (Fig. 3).

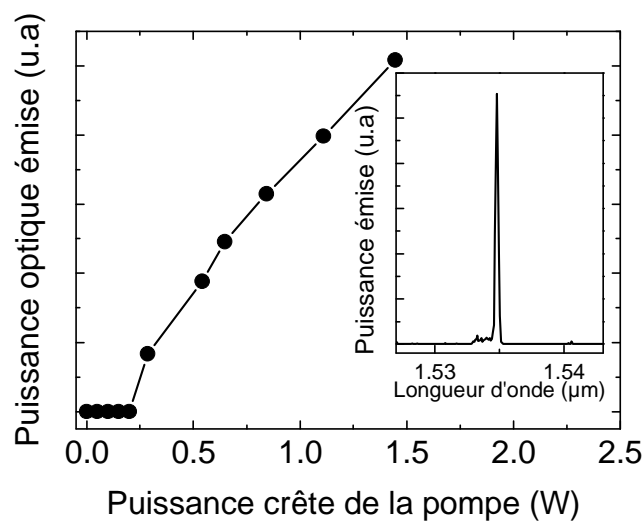

Fig 3 : Puissance optique émise en fonction de la puissance de pompe. En insert est présenté le spectre obtenu au niveau du seuil.

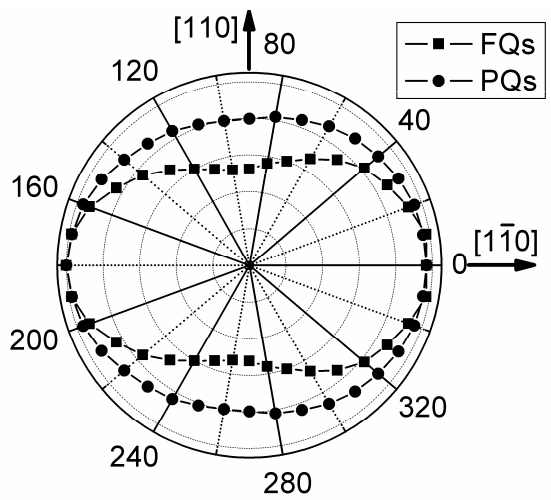

Fig 4 : Représentation du taux de polarisation (0\% au centre, $100 \%$ à l'extérieur) de la luminescence intégrée de FQs et de PQs par rapport à la direction [1-10]

L'étude plus approfondie d'un tel VCSEL montre qu'il existe deux états de polarisation selon deux directions cristallographiques bien particulières : [1-10] et [110]. Cette polarisation est variable selon les endroits sondés et elle est même susceptible de changement d'état au cours du fonctionnement du composant. Cette instabilité de la polarisation s'explique par la faible anisotropie du gain des PQs utilisées dans la zone active du VCSEL (Fig. 4). Dans le cas de structure quantiques se présentant sous forme de fils, l'anisotropie relevée en luminescence (Fig. 4) montre que l'orientation préférentielle des fils selon la direction [1-10] est plus favorable à une anisotropie du gain. En effet le rendement de luminescence dans la direction [1-10] est deux fois plus élevé que dans la direction [110]. L'introduction de telles structures quantiques en microcavité avec la méthode décrite précédemment va donc permettre de faire l'étude de nouveaux types de VCSEL et probablement améliorer la stabilité de la polarisation émise.

\section{4/ Conclusion}

Le développement d'un miroir de Bragg diélectrique $\left(\mathrm{a}-\mathrm{Si} / \mathrm{a}-\mathrm{SiN}_{\mathrm{x}}\right)$ à contraste d'indice élevé a permis d'obtenir un DBR performant. L'association d'une zone active à base de PQs réalisée en MBE avec deux miroirs a-Si/a$\mathrm{SiN}_{\mathrm{x}}$ a été rendue possible grâce à la technique de collage métallique. Le remplacement de PQs par des FQs dans la zone active introduira dans la cavité un matériau présentant une anisotropie de gain plus élevée ce qui stabilisera la polarisation émise par le VCSEL.

\section{Références :}

[1] T. Yoshikawa, H. Kosaka, M. Kajita, K. Kurihara, Y. Sugimoto and K. Kasahara, Electron. Lett. 31, 1573-1574 (1995)

[2] Y.L. Okuno, J. Geske, K.G. Gan, Y.J. Chiu, S.P. DenBaars and J.E. Bowers, Appl. Phys. Lett. 82, 2377-2379 (2003)

[3] T. Mukaihara, N. Ohnoki, Y. Hayashi, N. Hatori, F. Koyama, IEEE J. Sel. Topics Quantum Electron. 1, 667-673 (1995)

[4] Y. Ohno, H. Kanamori, S. Shimomura and S. Hiyamizu, Physica E 13, 892-895 (2002)

[5] A. Karim, S. Björlin, J. Piprek and J.E. Bowers, IEEE J. Sel. Topics Quantum Electron. 6, 1244-1253 (2000)

[6] C.C. Lee, C.Y. Wang and G. Matijasevic, IEEE Trans. Comp. Hybrids. Manuf. Technol. 16, 311 (1993). 